\title{
ОБЗОР АИТЕРАТУРЫ
}

DOI: https://doi.org/10.17816/psaic660

\section{СОВРЕМЕННЫЙ ВЗГАЯА НА ПРОБАЕМУ ОТВЕАЕНИЯ МОЧИ У АЕТЕЙ С ТЯЖЕАЫМИ НАРУШЕНИЯМИ МОЧЕИСПУСКАНИЯ}

\author{
(ㄱ) Р.О. Игнатьев ${ }^{1,2}$, Н.Б. Гусева ${ }^{1,2}$, М.А. Ромашин ${ }^{1}$
}

${ }^{1}$ Научно-исследовательский институт клинической хирургии, Федеральное государственное автономное образовательное учреждение высшего образования «Российский национальный исследовательский медицинский университет имени Н.И. Пирогова» Министерства здравоохранения Российской Федерации, Москва;

${ }^{2}$ Государственное бюджетное учреждение здравоохранения города Москвы «Детская городская клиническая больница № 9 им. Г.Н. Сперанского Департамента здравоохранения города Москвы», Москва

- Аля цитирования: Игнатьев Р.О., Гусева Н.Б., Ромашин М.А. Современный взгяя на проблему отведения мочи у детей с тяжелыми нарушениями мочеиспускания // Российский вестник детской хирургии, анестезиологии и реаниматологии. - 2020. - Т. 10. - № 2. C. 227-236. DOl: https://doi.org/10.17816/psaic660.

Мочеиспускание - сложный многокомпонентный процесс, нарушение которого зачастую ведет к серьезным последствиям со стороны мочевыделительной системы, а также к социальной дезадаптации и ухудшению качества жизни детей. Ввиду разнообразия состояний, ведущих к данным нарушениям и вовлечением в процесс нескольких систем (например, нервной системы при миелодисплазии), от врача требуются опыт и обширные знания в смежных областях. Хирургические способы отведения мочи имеют длинную историю. Хирурги всего мира в течение многих десятилетий пытаются решить данную проблему, и она до сих пор остается актуальной и не решенной. Соответственно большому разнообразию нарушений мочеиспускания существует внушительное количество различных типов и вариантов оперативных вмешательств, задача которых - коррекция не только анатомических и клинических нарушений, но и социально-психологическая адаптация ребенка. Хирург должен учитывать большое количество факторов: все нюансы патогенеза основного заболевания, сопутствующие патологии, конституциональные особенности ребенка, перспективы его социальной адаптации, семейную обстановку, в которой находится ребенок, готовность и нацеленность родителей на долгое и непростое лечение. Исходя из вышесказанного совершенно ясно, что отведение мочи у детей представляет многогранную проблему, требующую очень тщательного и индивидуального подхода к каждому пациенту для выбора оптимально метода лечения в каждом конкретном случае. От этого выбора зависит дальнейшая жизнь ребенка, его социальная адаптация, возможность получать образование и вести практически нормальный образ жизни. Цель настоящей работы - обзор и систематизация накопленных знаний по данной проблеме.

Ключевые слова: дети; хирургические методы отведения мочи; нарушение мочеиспускания; выбор тактики лечения.

\section{MODERN VIEW ON THE PROBLEM OF URINE DIVERSION IN CHILDREN WITH SEVERE URINARY DISORDERS}

\author{
(C) R.O. Ignatyev ${ }^{1,2}$, N.B. Guseva ${ }^{1,2}$, M.A. Romashin ${ }^{1}$
}

${ }^{1}$ Speranskiy Children's Municipal Hospital No. 9, Moscow, Russia;

${ }^{2}$ Pirogov Russian National Research Medical University

- For citation: Ignatyev RO, Guseva NB, Romashin MA. Modern view on the problem of urine diversion in children with severe urinary disorders. Russian J oumal of Pediatric Surgery, Anesthesia and Intensive Care. 2020;10(2):227-236. DOI: https://doi.org/10.17816/psaic660. 
Urination is a complex multicomponent process. Urinary disorders often lead to serious consequences on the urinary system, social maladaptation, and impairment of the quality of children's lives. Due to the large number of pathologies leading to these disorders and the involvement of several systems (for example, the nervous system in myelodysplasia), the doctor must be extensively knowledgeable and experienced in related fields. Surgical methods of urinary diversion have a long history. Surgeons worldwide have been trying to solve this problem for many decades, but it remains relevant and unsolved. The wide variety of urinary disorders has a correspondingly impressive number of types and options of surgical interventions. The goals of these surgical techniques are to correct the anatomical and clinical aspects of the disorders and assure the child's socio-psychological adaptation. Surgeons must consider many factors such as all the pathogenetic nuances of an underlying disease, comorbidities, the child's constitutional features, prospects for his or her social adaptation, the family home environment, parents' willingness to and focus on long and challenging treatment. Based on these details, it is evident that urinary diversion in children is a multifaceted problem. It requires a very careful and individualized approach to each patient to ensure choosing the optimal treatment method in each case. This choice will affect many aspects of the child's life, including his or her social adaptation, the ability to obtain an education and lead an almost normal lifestyle. The aim of this study is to review and systematize the accumulated knowledge on this subject.

Keywords: children; surgical methods of urine diversion; impaired urination; choice of treatment tactics.

Мочеиспускание, будучи лишь завершающей фазой весьма сложного многокомпонентного процесса накопления и выведения мочи, имеет тем не менее весьма важное значение для человека в плане формирования его социально-психологического статуса и качества жизни [1]. Говоря о пациентах детского возраста, нельзя не учитывать, что серьезные микционные нарушения, возникшие вследствие пороков развития, травм, опухолей или инвалидизирующих нейроинфекций, требуют не паллиативных решений, а более радикальных, отвечающих перспективам роста ребенка и его социализации [2]. К настоящему времени сложилось понимание, что анатомическая реконструкция нижних мочевых путей сама по себе не означает содружественное появление необходимой эвакуаторной функции. В этом и заключается основная сложность: любой известный способ моделирования части нижних мочевых путей из смежных органов или тканей не решает в полной мере специфическую задачу по обеспечению циклического процесса накопления и выведения мочи, поскольку порождает ряд других проблем $[3,4]$.

Главная цель хирургического лечения данной категории пациентов всегда одна: обеспечить эвакуацию мочи из организма. Способам ее достижения посвящены работы многих авторов $[1,2,5,6]$. Если она может быть достигнута несколькими способами, предпочтение отдают тому, который обеспечивает наименьшее количество осложнений и максимальное удобство в использовании. Здесь кроется очередная сложность, которая заключается в правильной оценке перспективы для ребенка. Если его двигательные и психические возможности допускают дальнейшую социализацию, то обеспечение наилучшей континенции становится более предпочтительным, чем удобство пользования и ухода [1]. Напротив, для ребенка в околовегетативном статусе удержание мочи принципиального значения не имеет, тогда как удобство доступа к стоме и простой уход за ней весьма желательны. Следовательно, выбирая стратегию в отношении ребенка-инвалида с тяжелыми нарушениями мочевыделения, специалист должен хорошо представлять принципы, лежащие в основе той или иной хирургической техники [5]. Для того чтобы разобраться в множестве существующих наработок по проблеме отведения мочи у детей, требуется их периодическая систематизация, что и послужило целью данной работы.

Как уже было сказано, бесперебойная эвакуация мочи из организма - это главная задача, которую можно решать как безоперационными, так и чисто хирургическими методами. Бесспорно, что технология чистой интермиттирующей катетеризации (CIC - clean intermittent catheterization), предложенная J. Lapides, в силу очевидности и относительной простоты долго будут считать золотым стандартом и у детей и у взрослых [4]. Гораздо менее очевидны критерии выбора методов хирургического отведения мочи ввиду большого количества модификаций и разноречивых данных об их эффективности 
и безопасности [2]. Так, одна из рабочих классификаций видов деривации мочи приведена ниже. Она достаточно удобна и используется на практике.

1. Накожное отведение мочи без использования кишечника:

a) аппендиковезикостомия - операция типа Митрофанова (Mitrofanoff);

б) надлобковая везикостомия с тубулярным или губовидным свищом;

в) уретерокутанеостомия;

г) пиелостомия;

д) нефростомия.

2. Отведение мочи в просвет кишечника:

a) простая уретеросигмостомия;

б) уретеросигмостомия с формированием резервуара из сигмовидной кишки - операции типа Mainz pouch II и Hassan.

3. Отведение мочи в изолированный кишечный кондуит без континенции («влажная» стома):

a) илеокондуит - операция Bricker;

б) колонкондуит - операция Mogg.

4. Отведение мочи в изолированный кишечный кондуит с континенцией («сухая» стома):
a) операция Kock-pouch;
б) операция Mainz pouch I;
в) операция Indiana pouch.

5. Ортотопическая пластика мочевого пузыря с отведением мочи по нативной уретре:

a) гастроцистопластика;

б) илеоцистопластика;

в) сигмоцистопластика;

г) ректоцистопластика

Редко используемый у детей, но важный с практической точки зрения, - метод чрескожной надлобковой цистостомии. Основанием для выбора данного вмешательства могут быть такие его достоинства, как:

- быстрота выполнения;

- минимальная травматичность;

- возможность проведения под местной анестезией вне операционной [7].

В развитых странах в педиатрической практике чрескожную цистостомию применяют ситуационно, в экстренных случаях. Вмешательство проводят под ультразвуковым контролем с использованием специальных одноразовых наборов. В некоторых клиниках (с недостаточным экономическим обеспечением) для пункционной цистостомии используют многоразовые металлические троакары, но это следует считать вынужденной мерой в экстремальных условиях, когда альтернати- вы нет [8]. Достоинство чрескожной цистостомии - это то, что при необходимости это вмешательство можно провести непосредственно у постели больного, если этого требует ситуация (например, у пациентов в отделении реанимации и интенсивной терапии) [9].

Уретерокутанеостомия представляет один из старейших методов деривации мочи. Суть ее заключается в простом выведении мочеточника в виде стомы на переднюю брюшную стенку или на кожу в поясничной области. На сегодня уретерокутанеостомия как самостоятельный способ длительной деривации мочи применяют относительно редко и по весьма ограниченным показаниям. Среди таковых следует упомянуть паллиативное отведение мочи у неоперабельных пациентов; анатомические особенности или патологические изменения, делающие выбор других методов невозможным; младенческий возраст в сочетании с врожденной обструктивной уропатией (как превентивный этап перед реконструктивным вмешательством). Плюсы уретерокутанеостомии заключаются в простоте и быстроте операции. Недостатки же представлены отсутствием континенции и частыми осложнениями в виде перистомического дерматита, инфекции верхних мочевых путей с развитием хронической почечной недостаточности [10].

Пиелостомия также редко используемый в наши дни способ длительного отведения мочи. При данном методе отток мочи осуществляют по эластичной трубке, установленной в просвет почечной лоханки и выведенной на поверхность тела. К показаниям для проведения пиелостомии относят: острый гнойный нефрит (с целью снижения внутрипочечного давления), уролитиаз с пиелонефритом, обструкция мочеточника различного генеза, туберкулез почки с нагноившимися очагами и ряд других [11]. Плюсы и минусы пиелостомии принципиально не отличаются от таковых при уретерокутанеостомии.

Чрескожная нефростомия также один из преимущественно «экстренных» методов. Впервые eе предложили Goodwin et al. в качестве мини-инвазивного вмешательства для декомпрессии верхних мочевых путей при их обструкции [43]. Нефростомия быстро нашла применение в урологии для временного отведения мочи и ее продолжают широко использовать. Процедура довольно проста и безопасна (осложнения возникают очень редко и летальность после чрескожной нефростомии составляет 0,05-0,3\%) [12]. При этом 
у детей нефростомию как самостоятельный способ продолжительного отведения мочи применяют очень ограниченно.

Гораздо более актуальным способом длительной деривации мочи является «влажная» везикостомия. К ней прибегают, когда консервативные мероприятия по восстановлению мочеиспускания не имеют должного успеха, в результате чего развивается уретерогидронефроз или некупируемый пиелонефрит [13]. Ее также применяют при невозможности осуществления CIC родителями, ухаживающим персоналом или самим ребенком, что актуально для детей из неблагополучных семей и детских домов. Обычно везикостомия является временной мерой по отведению мочи у детей раннего возраста с перспективой последующей ликвидации стомы или перевода ее в континентную форму. Наиболее известны технологии наложения везикостом, предложенные Blocksom и J. Lapides (прямая и обратная техники) (Николаев В.В.) [2]. Главные недостатки везикостомии - отсутствие континенции и высокая частота осложнений, именно поэтому данный метод в подавляющем большинстве случаев является временным [14]. К основным осложнениям относят стенозирование стомы, эвагинацию мочевого пузыря через стому, прогрессирующую инфекцию мочевыводящих путей.

Несколько иная идеология присуща операциям типа Bricker и Mogg, которые имеют целью отведение мочи в кишечный кондуит. Принцип этих операций состоит в деривации мочи в специально созданный резервуар, в качестве которого используют изолированный сегмент тонкого или толстого отделов кишечника соответственно. После имплантации в неокондуит одного или обоих мочеточников (современные модификации подразумевают антирефлюксную защиту) появляется возможность накопления мочи с последующим ее оттоком через специально сформированную стому. Последняя при таких видах вмешательств получается инконтинентной, то есть без механизма удержания. К сожалению, проверку временем эти операции прошли только у взрослых пациентов онкологического профиля, где их применяют главным образом после цистэктомии [15]. В детской практике операции типа Mogg/Bricker применяют редко, хотя вопрос об их применении у детей пристально изучался.

Достоинства этих операций состоят в отработанности техники и относительно не- сложном послеоперационном уходе. Список недостатков гораздо более существенный и включает (помимо всего прочего):

- наличие постоянно функционирующего мочевого свища, доставляющего пациенту как физический, так и психологический дискомфорт;

- потребность в постоянном внешнем резервуаре для сбора мочи (мочеприемнике); - возникновение рефлюкса мочи из кондуита в мочеточники с соответствующими последствиями (хроническая мочевая инфекция, образование камней, хроническая болезнь почек (ХБП) с утратой почечных функций).

Существуют методы отведения мочи в кишечник без нарушения его целостности, такие как классический вариант уретеросигмостомии и уретеросигмостомия с формированием резервуара из сигмовидной кишки по методикам Mainz pouch II и Hassan. Суть этих операций заключается в создании резервуара с низким давлением для накопления мочи, который представлен сигмовидной кишкой или участком толстой кишки, частично изолированным от кишечного тракта.

Классическая уретеросигмостомия была первой операцией, обеспечивающей больному противоестественное, но контролируемое мочеиспускание. Впервые ее выполнил Simon I. в 1852 г. В настоящее время данную операцию практически не применяют ввиду многочисленных осложнений, неизбежно возникающих в послеоперационном периоде. Основные из них: восходящая инфекция мочевых путей, ведущая к уросепсису и хронической почечной недостаточности, и тяжелые электролитные нарушения [16].

Более удачными оказались операции по формированию мочевого резервуара с низким давлением (Mainz pouch II и Hassan). Достоинствами подобных операций является то, что у пациента сохраняется возможность контролируемого мочевыведения за счет континентной функции анального сфинктерного комплекса без применения специальных приспособлений (катетеров, мешков для сбора мочи), нет внешних физических и косметических дефектов. Эти обстоятельства способствуют повышению качества жизни и облегчают социализацию пациента. Кроме того, детубуляризация участка кишки устраняет его перистальтику, тем самым создавая резервуар с низким давлением, что улучшает долгосрочный прогноз в отношении функцио- 
нальной сохранности верхних мочевых путей. Несмотря на многочисленные преимущества, есть и недостатки [17]. Пожалуй, основной из них - это смешение двух изначально раздельных продуктов жизнедеятельности (мочи и кала) и, как следствие, контаминация мочевых путей кишечной флорой. Не менее важный недостаток - это часто возникающий стеноз имплантированных в кишку мочеточников и прогрессирующий гидронефроз. Ко всему прочему, после данных операций у большинства больных отмечают развитие гиперхлоремического ацидоза, в связи с чем необходим регулярный прием ощелачивающих растворов и постоянный контроль кислотно-щелочного состояния крови. Еще один весомый недостаток - риск развития смешанного фекально-мочевого недержания. На протяжении всей жизни данные пациенты находятся в зоне высокого риска развития злокачественных опухолей в месте имплантации мочеточников [18].

Поиск альтернативных путей деривации мочи привел доктора Нильсона Кока в 1969 г. к новому методу. Суть его заключалась в создании артифициального мочевого пузыря из участка подвздошной кишки с выведением континентной стомы на переднюю брюшную стенку. Качественным отличием от методик Mainz pouch II и Hassan являлось то, что пассаж мочи и кала оставался раздельным. На сегодняшний день существует множество модификаций операций Kock pouch, основными и самыми признанными являются Mainz pouch I и Indiana pouch. Основное отличие этих модификаций заключается в используемом участке кишки для создания неоцистиca. Для достижения континенции используют: инвагинированный сегмент подвздошной кишки (Kock pouch), баугиниеву заслонку с пликацией сегмента подвздошной кишки (Mainz pouch I), приводящий сегмент подвздошной кишки с пликацией (Indiana pouch). Во всех вышеперечисленных операциях наиболее важные моменты - детубуляризация и реконфигурация кишечного сегмента для создания резервуара большой емкости с низким давлением, формирование клапана для удержания и защита верхних мочевых путей от рефлюкса [19].

Несомненное достоинство данных операций представляет достигаемая континенция и, как следствие, улучшение качества жизни пациентов. Раздельный пассаж мочи и кала (в отличие от операций Mainz pouch II и Hassan) также очень важен в отношении протекции верхних мочевых путей. Ввиду большого объема и низкого давления в неоцистисе, мочевой рефлюкс наблюдается достаточно редко, что также один из важнейших плюсов подобных операций. К недостаткам можно отнести техническую сложность и продолжительность вмешательств, высокую вероятность образования конкрементов в созданном резервуаре и вторичную диарею после резекции илеоцекального отдела кишечника [20].

Результатом дальнейшего развития континентного направления в реконструктивной урологии стала разработка нового метода деривации мочи - ортотопической пластики мочевого пузыря с мочеиспусканием по нативной уретре. Суть данных операций заключается в создании артифициального мочевого пузыря с помощью участка кишечника (или желудка) с имплантированными в него мочеточниками, которому придают ортотопическое положение, характерное для нативного мочевого пузыря, впоследствии создают соединение неоцистиса с уретрой. В итоге пациент, как и здоровый человек, осуществляет мочеиспускание через мочеиспускательный канал. В качестве пластического материала для нового «мочевого пузыря» используют различные участки желудочно-кишечного тракта: желудок, подвздошную, сигмовидную или прямую кишку, илеоцекальный сегмент, восходящую ободочную кишку. В настоящее время в подавляющем большинстве случаев предпочтение отдают подвздошной кишке, и самые распространенные операции - илеоцистопластика по Hautmann, по Studer, а также по Camey [21].

При любой модификации ортотопической цистопластики необходимо учитывать следующие моменты:

- кишечный сегмент должен быть достаточной длины для создания резервуара с большим объемом и низким давлением; - уретерокишечный анастомоз выполнять по антирефлюксной методике;

- выбор способа анастомозирования уретры и неоцистиса должен обеспечить сохранность рецепторных зон проксимальной уретры и сократительной функции сфинктерного комплекса.

Достоинства данной техники очевидны, главное из них в сравнении с вышеописанными операциями - мочеиспускание по нативной уретре, что значительно улучшает 
качество жизни пациентов. Большой объем неоцистиса с низким давлением и антирефлюксная имплантация мочеточников обеспечивают защиту верхних мочевых путей [22]. Среди недостатков можно выделить техническую сложность данных операций. Наиболее характерными поздними их осложнениями (собственно, как у и всех операций по созданию неоцистиса с помощью участка ЖКТ) это кишечная непроходимость, некроз резервуара, стеноз мочеточниковых соустий, метаболический ацидоз и илеофеморальный тромбоз. При этом частота развития осложнений относительно невелика и наблюдаются не более чем в $6 \%$ случаев (Studer et al., [44]).

Как видно из приведенных данных, хирургические методы отведения мочи не могут стать универсальным решением в большинстве клинических ситуаций в силу присущих им принципиальных недостатков. По-прежнему консервативный подход к решению проблемы неадекватного транспорта мочи в виде СІС является золотым стандартом [23-25]. Данную процедуру осуществляют различными катетерами: одноразовыми и многоразовыми, с лубрикантами и без. Исследования показывают, что использование многоразовых катетеров (в сравнении с одноразовыми) ассоциировано с большей частотой инфекции мочевыводящих путей $[26,27]$. СIC применяют при нейрогенных расстройствах мочевого пузыря на фоне миелодисплазии, травм спинного мозга и других состояниях, связанных с наличием остаточной мочи, соответственно, риском рефлюкса в верхние мочевые пути $[28,29]$. Введение этого метода в практику значительно снизило частоту таких осложнений, как ХБП, пиелонефрит, эрозивный цистит, конкременты и рак мочевого пузыря.

Несмотря на все достоинства, CIC в некоторых случаях имеет свои ограничения у детей с непроходимостью и сохранной чувствительностью уретры, конституциональными особенностями [30]. Отдельные исследования показывают ухудшение качества жизни пациентов в связи с переходом к СIC [31]. Следовательно, такая техника, при всех достоинствах - не универсальное решение проблемы, и определенный контингент больных нуждается в альтернативном способе отведения мочи.

Довольно удачной попыткой соединить достоинства различных подходов явилась разработка технологии создания континентных самокатетеризируемых стом. Впервые подобную операцию представил профессор Paul Mitrofanoff в 1980 году. Он предложил использовать аппендикс в качестве естественной трубчатой структуры, посредством которой можно соединять мочевой пузырь и поверхность кожи. Мобилизованный на сосудах собственной брыжейки червеобразный отросток одним концом анастомозируют с мочевым пузырем, а другой выводят на кожу с формированием небольшого губовидного свища [32]. В дальнейшем катетеризацию мочевого пузыря осуществляют через аппендикостому, которая (при соблюдении рекомендованной техники) обеспечивает континенцию мочи. В настоящее время все больше распространяется и развивается лапароскопия, в том числе в реконструктивной урологии у детей, и аппендиковезикостомию по Митрофанову это развитие не обошло стороной. Среди абсолютных преимуществ лапароскопической аппендиковезикостомии - меньшая травматичность операции, отличный косметический результат и сокращение сроков пребывания больного в стационаре [33-35]. Данная операция достаточно сложна технически, поэтому выполнять ее должен опытный хирург, обладающий хорошими навыками в области лапароскопии. Одними из новейших и весьма перспективных являются робот-ассистированные операции [36]. На данный момент такие операции делают в немногочисленных центрах, ввиду большой стоимости роботизированных аппаратов. Тем не менее существуют сообщения и о робот-ассистированной аппендиковезикостомии. Результаты таких операций не выявляют существенных различий в количестве острых послеоперационных осложнений и повторных операций, связанных с ними, в сравнении с открытыми операциями [37]. Но нельзя не учитывать очень малый опыт подобных операций, поэтому окончательные выводы делать слишком рано. Не вызывают сомнения такие достоинства робот-ассистированной аппендиковезикостомии, как меньшая инвазивность, вследствие чего происходит более быстрое восстановление и сокращение сроков пребывания в стационаре, меньшее применение анальгетиков в послеоперационном периоде и несомненно более выгодные косметические результаты по сравнению с открытой операцией $[38,39]$.

Подобные аппендиковезикостомии цели преследует операция по Янгу-Монти. Отли- 
чие состоит в том, что в качестве артифициального свищевого канала используют не аппендикс, а тубуляризованный участок тонкой кишки. Этот вид операции считается «второй линией» после аппендиковезикостомии по Митрофанову [30]. Необходимость подобной операции возникает при невозможности использования аппендикса в качестве кондуита. Такие ситуации возникают, когда пациенту уже была проведена аппендэктомия, длинна отростка не достаточна для создания адекватного соустья без натяжения, либо когда в ходе операции появляются проблемы мобилизации червеобразного отростка с нарушением его кровоснабжения.

Показания к формированию катетеризируемых континентных стом довольно немногочисленны. Среди них невозможность катетеризации через уретру в связи с ее недосягаемостью (вследствие выраженных деформаций скелета, ожирении, недостаточной подвижности), непроходимость уретры или et сохранная чувствительность и, соответственно, болезненность [5]. Некоторые пациенты с чисто эмоциональной точки зрения отмечают дискомфорт и снижение качества жизни в связи с CIC и даже испытывают отвращение к этому методу [40]. У них аппендиковезикостомия или операция Янга - Монти также представляет приемлемую альтернативу. Конечно, требуются дальнейшие исследования, которые более четко определят спектр показаний для подобных операций. Не исключено, что недооценка достоинств и недостатков метода связана с расширением перечня ситуаций, когда применяются такие вмешательства. Изначально метод Митрофанова был разработан для детей с нейрогенным мочевым пузырем, но на сегодняшний день его используют при многих других состояниях, таких как экстрофия мочевого пузыря, эписпадия, злокачественные новообразования таза, урогенитальный синус, травматические повреждения и стриктуры уретры [41]. Разумеется, при этом возникают предпосылки для более частого развития осложнений, таких как стомальная инконтиненция, эвагинация стомы, стеноз, перистомальная грыжа, конкременты мочевого пузыря, инфекции мочевыводящих путей [42]. Если тщательно рассмотреть риски и достоинства каждого метода на этапе дооперационного планирования, можно добиться неплохих результатов. В большинстве исследований авторы указывают на то, что в соответствующих клинических ситу- ациях мочевая континенция при создании катетеризируемых аппендиковезикостом была достигнута более чем в 90 \% случаев $[32,40,42]$.

Возвращаясь к исходному тезису, что первоочередной задачей любого вмешательства у рассматриваемой группы пациентов является обеспечение бесперебойной эвакуации мочи и выздоровление организма, можно развить его. Второй (в плане очередности, но не значимости) задачей мы считаем обеспечение приемлемого качества жизни ребенка, обязательно с учетом его перспектив роста и развития. Не вызывает сомнений, что далеко не все пациенты с тяжелыми нарушениями мочевыделения бесперспективны в плане социализации в современном обществе. После удачно проведенных вмешательств по деривации мочи дети могут легче социализироваться, получать образование, в дальнейшем создавать семьи и вести практически нормальный образ жизни.

Исходя из всего вышесказанного совершенно понятно, что на сегодняшний день существует множество различных методов отведения мочи. Для каждого из них есть различные показания и противопоказания. Выбор в каждой конкретной клинической ситуации определяют индивидуально для каждого пациента, исходя из этиологии и патогенеза основного заболевания, сопутствующих патологий и множества других факторов, в связи с чем систематизация накопленного опыта и периодическое представление результатов должны помочь хирургам лучше ориентироваться в проблеме.

\section{АОПОАНИТЕАЬНАЯ ИНФОРМАЦИЯ}

Конфликт интересов. Авторы данной статьи подтвердили отсутствие конфликта интересов и финансовой поддержки, о которых необходимо сообщить.

Conflict of interest. The authors of this article confirmed the lack of interest and financial support, which should be reported.

\section{АИТЕРАТУРА}

1. Данилов В.В, Данилов В.В. Нейроурология. В 3-х т. Т. 1- Владивосток: Клуб Директоров, 2019. - 280 c. [Danilov VV, Danilov VV. Neurourology. Vladivostok: Club Direktorov; 2019. Vol.1. 280 p. (In Russ.)]

2. Гусева Н.Б., Игнатьев Р.О. Миелодисплазия у детей. Организация и оказание специализи- 
рованной медицинской помощи. - М.: 2017. [Guseva NB, Ignat'ev RO. Mielodisplaziya u detej. Organizaciya i okazanie specializirovannoj medicinskoj pomoshchi. Moscow; 2017. (In Russ.)]

3. Державин В.М., Казанская И.В., Вишневский Е.Л., Гусев Б.С. Диагностика урологических заболеваний у детей. Библиотека практического врача. - М.: Медицина, 1984. - 210 с. [Derzhavin VM, Kazanskaya IV, Vishnevskii EL, Gusev BS. Diagnostika urologicheskih zabolevanij u detej. Biblioteka prakticheskogo vracha. Moscow: Meditsina; 1984. 210 p. (In Russ.)]

4. Вишневский Е.Л., Лоран О.Б., Вишневский А.Е. Клиническая оценка расстройств мочеиспускания. - M.: Терpa; 2001. 96 с. [Vishnevskii EL, Loran OB, Vishnevskii AE. Klinicheskaya ocenka rasstrojstv mocheispuskaniya. Moscow: Terra; 2001. 96 p. (In Russ.)]

5. Осипов И.Б., Красильников Д.Е., Соснин Е.В., и др. 15-летний опыт континентной везикостомии в лечении нейрогенного мочевого пузыря // Урологические ведомости. - 2016. - Т. 6. C. 79. [Osipov IB, Krasil'nikov DE, Sosnin EV, et al. 15-letnij opyt kontinentnoj vezikostomii $\mathrm{v}$ lechenii nejrogennogo mochevogo puzyrya. Urologicheskie vedomosti. 2016;6:79. (In Russ.)]

6. Николаев В.В., Степанов Э.А. Стриктуры уретры у детей. - М.: Медицина, 1998. [Nikolaev VV, Stepanov EhA. Urethral strictures in children. Moscow: Medicine; 1998. (In Russ.)]

7. Ibrahima D, Bouréima $\mathrm{O}$, Ibrahima $\mathrm{S}$, Aliou B. Cystostomie percutanée à la pince de Kelly: indications, technique et resultants. Pan African Medical Journal. 2015;22:164. DOI: https://doi. org/10.11604/pamj.2015.22.164.7280.

8. Muhammad AS, Agwu NP, AbdulwahabAhmed A, et al. Safety and efficacy of ultrasoundguided percutaneous suprapubic cystostomy in resource-poor setting: A 7-year review. Urol Ann. 2018;10(1):24-28. DOI: https://doi.org/10.4103/ UA.UA_104_17.

9. Luk YS, Shin JH, Khoo JL. Bedside ultrasoundguided percutaneous cystostomy in an infant in the neonatal intensive care unit. Ultrasonography. 2015;34(4):324-327. DOI: https://doi.org/10.14366/ usg. 14058.

10. Иванов В.В. Уретерокутанеостомия в неотложной урологии // Бюллетень ВСНЦ СО РАМН. 2008. - № 3(61). - C. 80-81. [Ivanov VV. Ureterocutaneostomy in emergency urology. Bulletin of SNTs SO RAMN. 2008;3(61):80-81. (In Russ.)]

11. Immergut MA, Jacobson JJ, Culp DA, Flocks RH. Cutaneous Pyelostomy. J Urol. 1969; 101(3):276279. DOI: https://doi.org/10.1016/S00225347(17)62328-0.
12. Turo R, Horsu S, Broome J, et al. Complications of percutaneous nephrostomy in a district general hospital. Turk J Urol. 2018;44(6):478-483. DOI: https://doi.org/10.5152/tud.2018.37336.

13. Dönmez M, Carrasco A, Saltzman AF, et al. Long-term outcomes of cutaneous vesicostomy in patients with neuropathic bladder caused by spina bifida. J Pediatr Urol. 2017;13(6):622. DOI: http:// doi.org/10.1016/j.jpurol.2017.05.015.

14. Sharifiaghdas F, Mirzaei M, Nikravesh N. Can transient resting of the bladder with vesicostomy reduce the need for a major surgery in some patients? J Pediatr Urol. 2019;15(4):379. DOI: http://doi.org/10.1016/j.jpurol.2019.03.026.

15. Спицын И.М., Перепечай В.А., Коган М.И. Гетеротопические континентные формы деривации мочи // Вестник урологии. - 2017. - Т. 5. № 1. C. 64-70. [Spitsin IM, Perepechay VA, Kogan MI. Continent heterotopic urine derivation forms. Urology Herald. 2017;5(1):64-70. (In Russ.)] DOI: https://doi. org/10.21886/2306-6424-2017-5-1-64-70.

16. Zimmer V, Lammert F. Ureterosigmoidostomy. Dig Liver Dis. 2019;51(11):1618. DOI: https://doi. org/10.1016/j.dld.2019.08.026.

17. Bao J, Yue Z, Wu G, et al. Technique and results in total laparoscopic radical cystectomy with sigmoidorectal pouch (Mainz pouch II) - an initial experience. Exp Ther Med. 2017;13(5):17491752. DOI: https://doi.org/10.3892/etm.2017.4163.

18. D'elia G, Pahernik S, Fisch M, et al. Mainz Pouch II technique: 10 years' experience. BJU International. 2004;93(7):1037-1042. DOI: https:// doi.org/10.1111/j.1464-410x.2003.04777.x.

19. Khalil F, Fellahi S, Ouslim H, et al. Long-term follow-up after ileocaecal continent cutaneous urinary diversion (Mainz I pouch): A retrospective study of a monocentric experience. Arab J Urol. 2015;13(4):245-249. DOI: https://doi.org/10.1016/j. aju.2015.09.004.

20. Chowdhary SK, Rao Katargadda LN, Kandpal DK, et al. Indiana pouch in children: A 15-year experience. J Pediatr Urol. 2014;10(5):911-916. DOI: https://doi.org/10.1016/j.jpurol.2014.02.009.

21. Chang Dwayne TS, Lawrentschuk N. Orthotopic neobladder reconstruction. Urol Ann. 2015;7(1):1-7. DOI: https://doi.org/10.4103/0974-7796.148553.

22. Malioris A, Dimitriadis G, Kampantais S, et al. Comparison Between Ambulatory and Conventional Urodynamics of the Modified Orthotopic Hautmann Neobladder. Int Neurourol J. 2015;19(4):265-271. DOI: https://doi.org/10.5213/ inj.2015.19.4.265.

23. Biardeau $\mathrm{X}$, Corcos J. Intermittent catheterization in neurologic patients: Update on genitourinary 
tract infection and urethral trauma. Ann Phys Rehabil Med. 2016;59(2):125-129. DOI: https:// doi.org/10.1016/j.rehab.2016.02.006.

24. Di Benedetto P. Clean intermittent selfcatheterization in neurourology. Eur J Phys Rehabil Med. 2011;47(4):651-659.

25. Prieto J, Murphy CL, Moore KN, Fader M. Intermittent catheterisation for long-term bladder management. Cochrane Database Syst Rev. 2017;8:CD006008. DOI: https://doi.org/ 10.1002/14651858.CD006008.pub4.

26. Saadat SH, Shepherd S, Van Asseldonk B, Elterman DS. Clean intermittent catheterization: Single use vs. reuse. Can Urol Assoc J. 2019;13(2):6469. DOI: https://doi.org/10.5489/cuaj.5357.

27. Pickard R, Chadwick T, Oluboyede $\mathrm{Y}$, et al. Continuous low-dose antibiotic prophylaxis to prevent urinary tract infection in adults who perform clean intermittent self-catheterisation: the AnTIC RCT. Health Technol Assess. 2018;22(24):i102. DOI: https://doi.org/10.3310/hta22240.

28. Assis GM, Faro ACM. [Clean intermittent self catheterization in spinal cord injury (in Brasil)]. Rev Esc Enferm USP. 2011;45(1):289-293. DOI: https:// doi.org/10.1590/s0080-62342011000100041.

29. Fumincelli L, Mazzo A, Martins JCA, et al. Quality of life of patients using intermittent urinary catheterization. Rev Lat Am Enfermagem. 2017;25:e2906. DOI: https://doi.org/10.1590/15188345.1816 .2906

30. Красильников Д.Е. Принцип Митрофанова в реконструктивной урологии. Обзор литературы // Педиатр. - 2010. - Т. 1. - № 1. C. 72-79. [Krasilnikov DE. Mitrofanoff principle in urinary tract reconstruction. Literature review. Pediatr. 2010;1:72-79. (In Russ.)]

31. Weynants L, Hervé F, Decalf V, et al. Clean Intermittent Self-Catheterization as a Treatment Modality for Urinary Retention: Perceptions of Urologists. Int Neurourol J. 2017;21(3):189-196. DOI: https://doi.org/10.5213/inj.1734824.412.

32. Iqbal N, Syed OZ, Bukhari AH, et al. Utility of Mitrofanoff as bladder draining tool: A single center experience in pediatric patients. Turk J Urol. 2018;45(1):42-47. DOI: https://doi.org/10.5152/ tud.2018.86836.

33. Nerli RB, Reddy M, Devraju S, et al. Laparoscopic mitrofanoff appendicovesicostomy: Our experience in children. Indian J Urol. 2012;28(1):28-31. DOI: https://doi.org/10.4103/0970-1591.94951.
34. Costa P, Ferreira C, Bracchitta D, Bryckaert PÉ. Laparoscopic appendicovesicostomy and ileovesicostomy: A step-by-step technique description in neurogenic patients. Urol Ann. 2019;11(4):399-404. DOI: https://doi.org/10.4103/ ua.ua_167_18.

35. Reddy MN, Nerli RB, Patil RA, Jali SM. Laparoscopic Mitrofanoff continent catheterisable stoma in children with spina bifida. Afr J Paediatr Surg. 2015;12(2):126-130. DOI: https://doi. org/10.4103/0189-6725.160356.

36. Howe A, Kozel Z, Palmerc L. Robotic surgery in pediatric urology. Asian J Urol. 2017;4(1):55-67. DOI: https://doi.org/10.1016/j.ajur.2016.06.002.

37. Grimsby GM, Jacobs MA, Gargollo PC. Comparison of Complications of Robot-Assisted Laparoscopic and Open Appendicovesicostomy in Children. J Urol. 2015;194(3):772-776. DOI: https://doi.org/10.1016/j.juro.2015.02.2942.

38. Chung PH, De S, Gargollo PC. Robotic appendicovesicostomy revision in children: description of technique and initial results. J Endourol. 2015;29(3):271-275. DOI: https://doi. org/10.1089/end.2014.0550.

39. Nguyen HT, Passerotti CC, Penna FJ, et al. Robotic assisted laparoscopic Mitrofanoff appendicovesicostomy: preliminary experience in a pediatric population. J Urol. 2009;182(4):15281534. DOI: https://doi.org/10.1016/j. juro.2009.06.055.

40. Levy ME, Elliott SP. Reconstructive techniques for creation of catheterizable channels: tunneled and nipple valve channels. Transl Androl Urol. 2016;5(1):136-144. DOI: https://doi.org/10.3978/j. issn.2223-4683.2016.01.04.

41. Sunada T, Kamido S, Hamada A, et al. Urinary Diversion Using an Appendicovesicostomy for Idiopathic Urethral Stricture: A Case Report. Hinyokika Kiyo. 2016;62(9):479-482. DOI: https:// doi.org/10.14989/ActaUrolJap_62_9_479.

42. Solanki S, Babu MN, Jadhav V, et al. Continent catheterizable conduit for urinary diversion in children: Applicability and acceptability. Afr J Paediatr Surg. 2015;12(1):33-35. DOI: https:// doi.org/10.4103/0189-6725.150965.

43. Dagli M, Ramchandani P Percutaneous nephrostomy: technical aspects and indications. Semin Intervent Radiol. 2011;28(4):424-437. DOI: https://doi.org/10.1055/s-0031-1296085.

44. Studer UE, Turner WH. The ileal orthotopic bladder. Urology. 1995 Feb;45(2):185-9. DOI: https://doi.org/10.1016/0090-4295(95)80002-6. 


\section{Информация об авторах}

Роман Олегович Игнатьев - д-р мед. наук, главный научный сотрудник отдела хирургии детского возраста, НИИ клинической хирургии. РНИМУ им. Н.И. Пирогова Минздрава России, Москва; заведующий отделом нейроурологии и урологии-андрологии, ГБУЗ «Детская городская клиническая больница № 9 им. Г.Н. Сперанского ДЗМ», Москва. E-mail: romarion74@mail.ru. ORCID iD: https://orcid.org/0000-0002-6393-1326.

Наталья Борисовна Гусева - д-р мед. наук, главный научный сотрудник отдела хирургии детского возраста НИИ клинической хирургии, РНИМУ им. Н.И. Пирогова Минздрава России, Москва; руководитель центра урологии, андрологии и патологии тазовых органов, ГБУЗ «Детская городская клиническая больница № 9 им. Г.Н. Сперанского ДЗМ», Москва. E-mail: gusevan-b@yandex.ru.

Максим Александрович Ромашин - клинический ординатор. ФГБОУ ВО РНИМУ им. Н.И. Пирогова Минздрава России, Москва. E-mail: romashinmaxim17@ yandex.ru. ORCID iD: https://orcid.org/0000-0003-34809812.
Information about the authors

Roman O. Ignatyev - Dr Sci. (Med.), Chief researcher of the department of pediatric surgery research Institute of clinical surgery. Pirogov Russian National Research Medical University (RNRMU), Moscow, Russia; Head of the Department of Neurology and Urology-Andrology, Speranskiy Children's Municipal Hospital No. 9 DZM, Moscow, Russia. E-mail: romarion74@yandex.com. ORCID iD: https://orcid.org/0000-0002-6393-1326.

Natal'ya B. Guseva - Dr Sci. (Med.), Chief researcher of the department of pediatric surgery research Institute of clinical surgery Pirogov Russian National Research Medical University (RNRMU), Moscow, Russia; chief of the center of urology, andrology and pathology of the pelvic organs, Speranskiy Children's Municipal Hospital No. 9 DZM, Moscow, Russia. E-mail: guseva-n-b@yandex.ru. ORCID iD: https://orcid.org/0000-0002-1583-1769.

Maksim A. Romashin - clinical resident. Pirogov Russian National Research Medical University (RNRMU), Moscow, Russia. E-mail: romashinmaxim17@yandex.ru. ORCID iD: https://orcid.org/0000-0003-3480-9812. 